\title{
TOWN HOUSE AND THE CITY: THE SEARCH FOR BEAUTY: LOOK UP!
}

\author{
PETR KALOUDA
}

Brno University of Technology, Faculty of Architecture, Poříćí 5, 63900 Brno, Czech Republic Tutor: doc. Ing. arch. Josef Kiszka

Theme of the dissertation: The town house and its influence on its surroundings, perpetuality and temporality

petr.kalouda@seznam.cz

\begin{abstract}
The topic is a quantitative analysis of the subjective perception of a town house and its visual factor. Its direct influence on the environment, affecting the locality and passers-by was observed. The focus is on the evaluation of the exterior and a correlation with its function and the suitability of the investment. The respondent group is composed of professionals and laymen. The correlation between the visual factor of a Town house, the attractiveness of areas and the house was unveiled.
\end{abstract}

KEY WORDS: town house, metropolitan house, satisfaction component, facade, building aesthetic, Brno

\section{Introduction}

The focus of this paper is finding the connections between the visual factor and the overall evaluation of a Town house in general. The city is an image of society. Town houses, with a few exceptions, are composed of simple facades with non complex windows. Significant places - formed by the presence of unique facades, according to artistic principles (Sitte, 2012), (Gehl, 2012) - are missing. The research aims to get new insights into sensitive and economically profitable development that can be used in practice. The pilot phase of a survey that serves to optimize the survey questionnaire, was performed. Town house we understand as a variation of a multi-storey building in or near the city centre. We investi- 
gate the influence of the aesthetic values of facades on the residents and passers-by.

Regarding the current state of knowledge, the relationship between satisfaction factors and the whole evaluation of the town houses was investigated in the initial study in Dhaka (Mridha, 2015). We conducted a conceptual replication (Bonnet, 2012) of the former study (Mridha, 2015), and we extended the study by adding visual factor evaluation. The author of the previous study claims that a closer examination of architectural elements has been neglected.

Considering the context, current development projects do not usually contain any major systematized use of architectural language in general. A sensitive approach to context is not present. The modernist context and continuity with the Brno functionalist architectural style is being used as an excuse for poor visual quality. The current approach of sensitivity to context surrounding buildings, seems not to be fully applied in practice. As Gehl mentioned, human scale is not always present (Gehl 2012). The major limiting factors of a project are its financial expenses. 'By applying the previous aspect of aesthetics to architectural research, we found evidence that architects and non-architects differ in their building preference... - "Studies have shown that differences exist between expert architects and laypeople in their aesthetic preferences” (Šafárová, 2019).

According to the current building development trend, the profitability of a house project cannot be neglected. Financial savings for heating are monitored. (Vyhl.č. 264 [National Regulation no. 264], 2020). The External Thermal Insulation Composite Systems is being used due to its relatively low price. "EIFS / ETICS" (Terraco EIFS, 2021) Polystyrene and mineral materials are being used. The shape of a house is usually restricted to a simple mass due to work processes. More complex shapes lead to higher economic demands in the construction process, and the house having higher energy consumption. Nevertheless, our hypothesis suggests that building aesthetics (street facades) directly influence the quality of public space. 'The nature of social activities varies depending on the context in which they occur" (Gehl, 2010).

\section{Methodology}

The original research proposal consisted of both factor analysis and regression analysis. Because of the limited respondent count, only the regression analysis was selected. An accurate definition of a Town House was created.

\subsection{Research project}

\subsubsection{Research goal}

How to design town houses (multifunctional building) in a city. To enable economical 
profitability, conserve functional and visual qualities, and increase the value of its surroundings.

\subsubsection{Research question}

How does the visible exterior of a town house relate to its evaluation?

\subsubsection{Hypothesis}

(1) Passers-by are actively interested in the exterior of town houses.

(2) The exterior of the town house directly affects its rating.

(3) The exterior of the house directly affects the evaluation of its surroundings.

(4) The exterior of the house is one of the keys of investment decisions.

\subsection{Research design}

The study investigated housing satisfaction in its socio-psychological context. Its design is based on the previous study Living in an apartment (Mridha, 2015). Both studies make correlational conclusions instead of causal conclusions. Correlation analysis was intended to reveal the relationship between subjective evaluation of the town house facades and its quantifiable parameters.

\subsection{Sampling}

(1) Respondent-driven sampling: Snowball sampling by e-mail. The possibility of nominating other research participants by the respondents themselves was enabled.

(2) Clustered sampling: To obtain data, groups of experts and practising architects on social networks were selected.

(3) Convenience sampling: To obtain data from non-professionals, groups on social networks, composed of laymen, were selected.

\subsection{Participants}

A total of 136 people agreed to participate. No one was excluded. See Fig 1: Sample characteristics

\subsection{Measurement instrument}

An anonymous online questionnaire was created to repeat a previous study. Data were collected for a period of 14 days. The key parts of the questionnaire were Current housing evaluation, evaluation of town houses facades, and overall evaluation of town houses. A total of 15 questions were defined. The language of the questionnaire (and native language of respondent group) was Czech. Data were then translated to English for the purpose of this study. The form uses the scale of the answers from 1 (worst) to 5 (best). 


\subsection{The components of satisfaction}

The following factors were selected and defined: Architectural features, Close surroundings of building, Apartment layout, Public Transportation, Car parking, Leisure and Recreation facilities, Real estate fees, Neighbourhood community, Management and maintenance, and Ambient Environment.

\section{Results}

See Fig 2: Data input part 1

See Fig 3: Data input part 2

\subsection{Data cross analysis}

Most respondents $(38,24 \%)$ live near the city centre, the least respondents live in individual housing located in the city outskirts where the urban sprawl process may be seen $(5,15 \%)$ followed by the city centre $(8,82 \%)$. The largest part of the sample consists of people aged 21-30 living near the city centre(14,71\%). The most common response to overall satisfaction with current housing was 4 and 5 points. The most satisfied group was those aged $71-80$. The least satisfied group was those from 11-20. The overall satisfaction of male respondents was slightly higer than that of female respondents.

See Fig 4: Data Cross analysis

\subsection{Regression analysis}

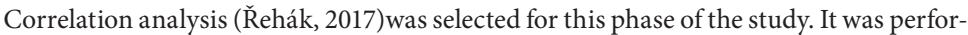
med in three areas of interest.

\subsubsection{Correlation analysis A - Current housing: Overall satisfaction and its components}

These values serve as reference numbers. We can see a correlation expressed by Spear-

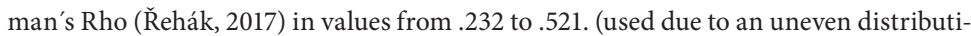
on of results). A higher count means higher existence of mutual relations. Mainly positive correlations were revealed. Overall satisfaction correlates the most with the layout of the apartment (.351), in second place are architectural elements (.296). A negative correlation of -.055 was found in connection with the availability of public transport.

See Fig 5: Correlation analysis

See Fig 8: Network analysis 


\subsubsection{Correlation analysis D, E - Town House: Appearance and com- ponents of residential satisfaction}

Analysis reveals stronger correlations than the previous part. The values of significant correlation are in the range of Spearmens rho from .246 to .540. We see a negative correlation between the evaluation of the facade and public transport (-.211), the availability of parking (-.126) and the availability of recreation and leisure (-.196).

See Fig 6: Correlation analysis

See Fig 9: Network analysis

\subsubsection{Correlation analysis D, F - Town House: Suitability of the in- vestment and Components of Residential Satisfaction}

This part uses the same variables as the second phase but evaluates the suitability of the investment. Therefore it shows significantly different values. The number of negative correlations is smaller (facade rating with public transport -.133). Significant correlations are found in the range from .252 to 0.535 and their total count is smaller. There is a significant correlation between "Facade affects area evaluation" And "Facade affects building evaluation" (.388) and strong correlation between "Management and maintenance" and "ambient environment"(.438), "Leisure and Recreation facilities" correlated to "Real estate fees".401.

See Figure 7: Correlation analysis

See Figure 10: Network analysis

\section{Discussion}

The study investigated housing satisfaction in its socio-psychological context. Its design is based on the previous study Living in an apartment (Mridha, 2015). Both studies make correlational conclusions instead of causal conclusions. Correlation analysis was intended to reveal the relationship between subjective evaluation of the town house facades and its quantifiable parameters.

\section{Conclusions}

The study investigated housing satisfaction in its socio-psychological context. Its design is based on the previous study Living in an apartment (Mridha, 2015). Both studies make correlational conclusions instead of causal conclusions. Correlation analysis was intended to reveal the relationship between subjective evaluation of the town house facades and its quantifiable parameters. 


\section{References}

MRIDHA, Mozammel. Living in an apartment. Journal of Environmental Psychology. Amsterdam: Elsevier, 2015, 43, 42-54. ISSN 0272-4944. Dostupné z: doi: https:// doi.org/10.1016/j.jenvp.2015.05.002

BONNET, Douglas. Replication-Extension Studies. Curr. Dir. in Psychol. Sci. 2012, 21(6), 409/412. Dostupné z: doi: https://doi.org/10.1177/0963721412459512

ŘEHÁK, Jan, 2017. Koeficienty pořadové korelace. [Sequence correlation coefficients] In: Sociologická enycklopedie (online). 11. 12. 2017 (cit. 11.9.2021). Dostupné z: https:// encyklopedie.soc.cas.cz/w/Koeficienty_po\%C5\%99adov\%C3\%A9_korelace

ŠAFÁROVÁ, Katarína, Martin PÍRKO, Vojtěch JUŘÍK, Tomáš PAVLICA a Ondřej NÉMETH. Differences between young architects' and non-architects' aesthetic evaluation of buildings. Frontiers of Architectural Research. Amsterdam: Elsevier, 2019, 8(2), 229-237. ISSN 2095-2635. Dostupné z: doi: https://doi.org/10.1016/j. foar.2019.04.001

SITTE, Camillo. Stavba měst podle uměleckých zásad. 2. české vyd. Brno: ÚÚR, 2012. ISBN 978-80-87318-21-8.

GEHL, Jan. Města pro lidi [Cities for people]. Brno: Partnerství, c2012. ISBN 978-80260-2080-6.

GEHL, Jan. Život mezi budovami: užívání veřejných prostranství [Life Between Buildings: Using Public space]. Boskovice: Albert, 2000. ISBN 80-85834-79-0.

Vyhláška č. 264/2020 Sb. o energetické náročnosti budov [National Regulation of Energy Consumption of the Building no. 264/2020] 
IO $^{\text {th }}$ ACAU 2021 conference papers

\section{Figure 1: Sample characteristics}
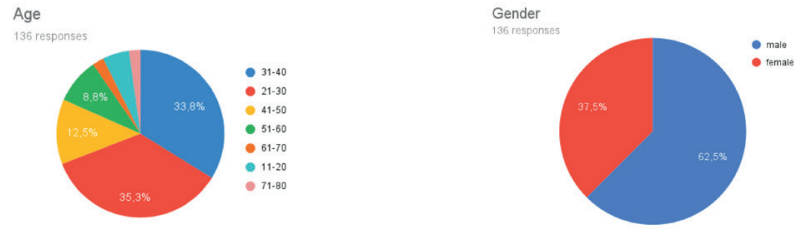

Educational attainment 136 responses
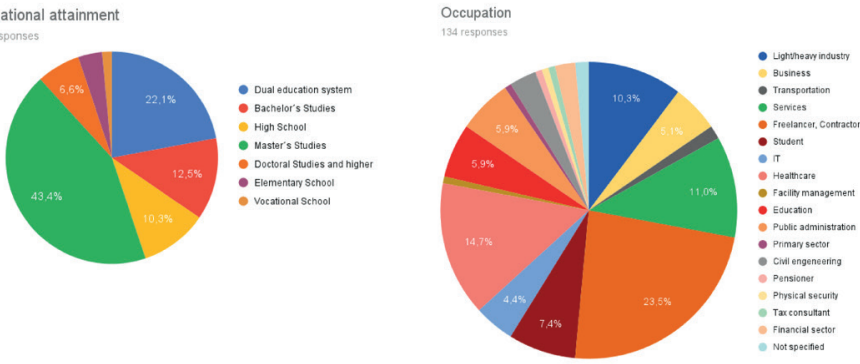

Type of current housing 136 responses

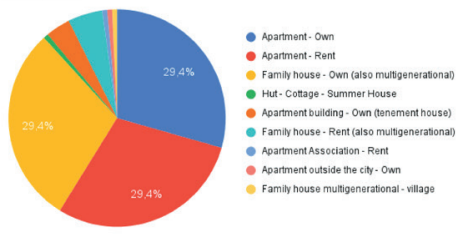

Location of current housing

136 responses

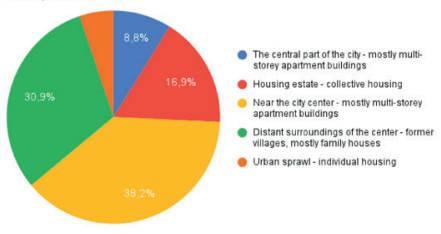


Petr Kalouda

\section{Figure 2: Data input, part 1}

Current housing satisfaction

136 responses

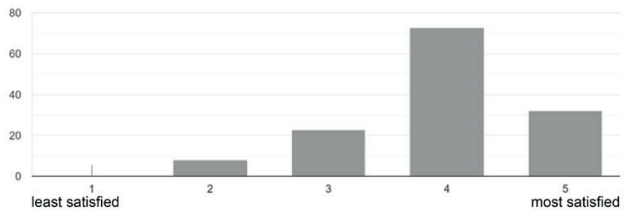

Factors of current housing satisfaction

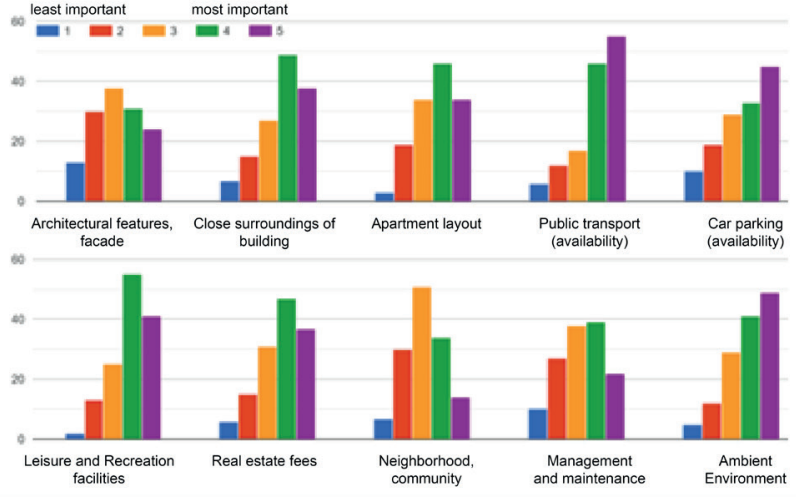

Frequency of movement in the central area of the city

136 responses

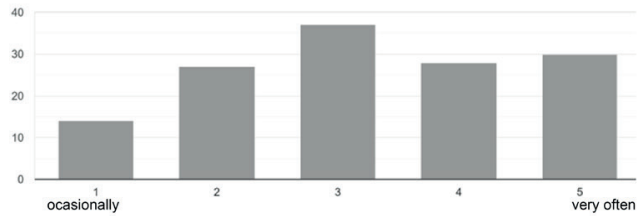

Mode of transport used when moving around the city

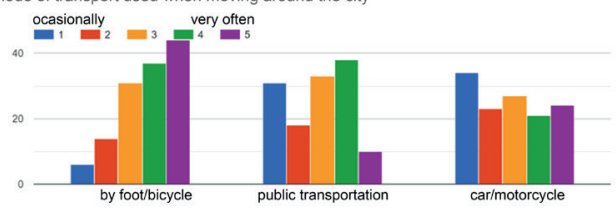


Figure 3: Data input, part 2

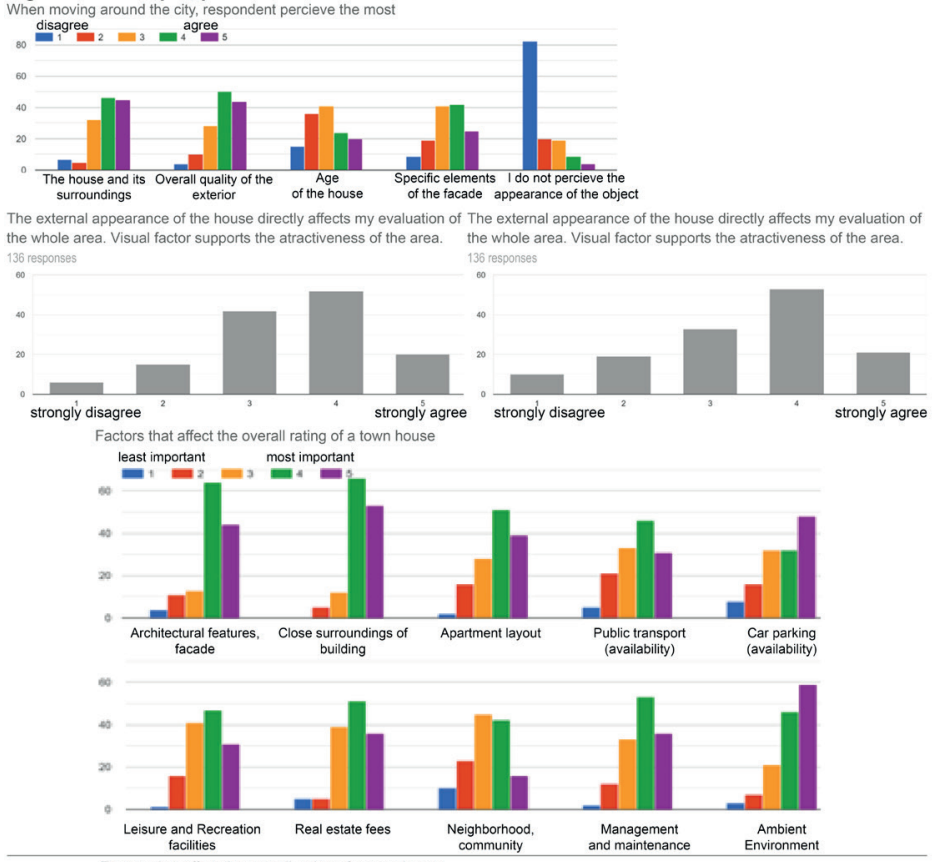

Factors that affect the overall rating of a town house
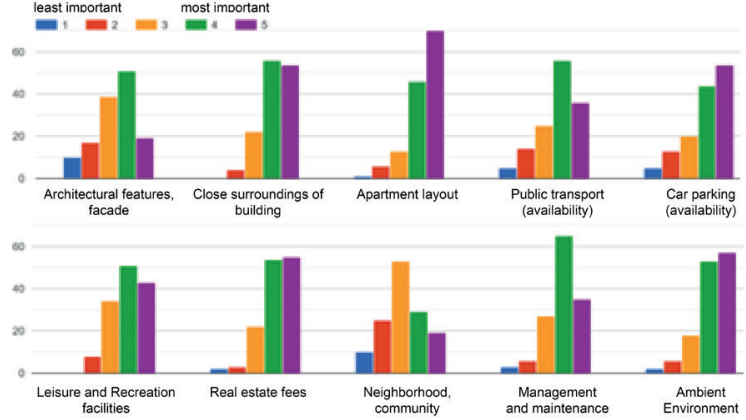


\section{Figure 4: Data cross analysis}

Cross analysis: Location of Current Housing and Age

\begin{tabular}{|c|c|c|c|c|c|c|c|c|}
\hline COUNTA of LOCATION OF HOUSING & AGE & & & & & & & \\
\hline LOCATION OF HOUSING & $11-20$ & $21-30$ & $31-40$ & $41-50$ & $51-60$ & $61-70$ & $71-80 \mathrm{C}$ & Celkovy součet \\
\hline Distant surroundirgs of the center - former villages, mostly farnily houses & $2.21 \%$ & $6.62 \%$ & $10.29 \%$ & $735 \%$ & $1.47 \%$ & $1.47 \%$ & $1.47 \%$ & $30.88 \%$ \\
\hline Housing estate - collective housing & $0.74 \%$ & $8.82 \%$ & $3.68 \%$ & $0.74 \%$ & $2.21 \%$ & $0.00 \%$ & $0.74 \%$ & $16.91 \%$ \\
\hline Near the city center - mostly multi-storey apartment buildings & $1.47 \%$ & $14.71 \%$ & $13.97 \%$ & $3.68 \%$ & $3.68 \%$ & $0.74 \%$ & $0.00 \%$ & $38.24 \%$ \\
\hline The central part of the cily - mostly multi-storey apartment buildings & $0.00 \%$ & $4.41 \%$ & $3.68 \%$ & $0.00 \%$ & $0.74 \%$ & $0.00 \%$ & $0.00 \%$ & $8.82 \%$ \\
\hline Urban spraw - individual housing & $0.74 \%$ & $0.74 \%$ & $221 \%$ & $0.74 \%$ & $0.74 \%$ & $0.00 \%$ & $0.00 \%$ & $5.15 \%$ \\
\hline Celkorỳ součat & $5.15 \%$ & $35.29 \%$ & $33.82 \%$ & $12.50 \%$ & $8.82 \%$ & $2.21 \%$ & $2.21 \%$ & $100.00 \%$ \\
\hline
\end{tabular}

\begin{tabular}{|c|c|c|c|c|c|}
\hline \multirow{2}{*}{$\begin{array}{l}\text { COUNTA of A: CURRENT HOUSING SATISFACTION } \\
\text { AGE }\end{array}$} & \multicolumn{5}{|c|}{ A: CURRENT HOUSING SATISFACTION } \\
\hline & 2 & 3 & 4 & 5 & Celkovy soudet \\
\hline $11-20$ & $14.29 \%$ & $14.29 \%$ & $57.14 \%$ & $1429 \%$ & $100.00 \%$ \\
\hline $21-30$ & $6.25 \%$ & $20.83 \%$ & $50.00 \%$ & $22.92 \%$ & $100.00 \%$ \\
\hline 31-40 & $8.70 \%$ & $13.04 \%$ & $56.52 \%$ & $21.74 \%$ & $100.00 \%$ \\
\hline $41 \cdot 50$ & $0.00 \%$ & $11.76 \%$ & $47.06 \%$ & $41.18 \%$ & $100.00 \%$ \\
\hline $51 \cdot 60$ & $0.00 \%$ & $25.00 \%$ & $75.00 \%$ & $0.00 \%$ & $100.00 \%$ \\
\hline $61-70$ & $0.00 \%$ & $33.33 \%$ & $30.33 \%$ & $33.33 \%$ & $100.00 \%$ \\
\hline $71-80$ & $0.00 \%$ & $0.00 \%$ & $33.33 \%$ & $66.67 \%$ & $100.00 \%$ \\
\hline Celkorý soucét & $5.88 \%$ & $16.91 \%$ & $53.68 \%$ & $23.53 \%$ & $100.00 \%$ \\
\hline
\end{tabular}

Cross analysis: Gender and Overall Current Housing Satisfaction

\begin{tabular}{|c|c|c|c|c|c|}
\hline \multirow{4}{*}{$\begin{array}{l}\text { COUNTA of A: CURRENT HOUSING SATISFACTION } \\
\text { GENDER }\end{array}$} & \multicolumn{5}{|c|}{ A: CURRENT HOUSING SATISFACTION } \\
\hline & 2 & 3 & 4 & & ysoucet. \\
\hline & $7.06 \%$ & $14.12 \%$ & $54.12 \%$ & $24.71 \%$ & $100.00 \%$ \\
\hline & $3.92 \%$ & $21.57 \%$ & $52.94 \%$ & $21.57 \%$ & $100.00 \%$ \\
\hline kory soućet & $5.88 \%$ & $16.91 \%$ & $53.68 \%$ & $23.53 \%$ & $100.00 \%$ \\
\hline
\end{tabular}

1 -male, 2 - femal

Cross analysis: Occupation and Overall Current Housing Satisfaction

\begin{tabular}{|c|c|c|c|c|c|}
\hline \multirow{2}{*}{$\begin{array}{l}\text { COUNTA OF A: CURRENT HOUSING SATISFACTION } \\
\text { OCCUPATION }\end{array}$} & \multicolumn{5}{|c|}{ A: CURRENT HOUSING SATISFACTION } \\
\hline & 2 & 3 & 4 & & y součet \\
\hline Business & $14.29 \%$ & $42.86 \%$ & $28.57 \%$ & $14.29 \%$ & $100.00 \%$ \\
\hline Civi engenering & $25.00 \%$ & $0.00 \%$ & $75.00 \%$ & $0.00 \%$ & $100.00 \%$ \\
\hline Education & $0.00 \%$ & $12.50 \%$ & $62.50 \%$ & $25.00 \%$ & $100.00 \%$ \\
\hline Facilty management & $0.00 \%$ & $0.00 \%$ & $100.00 \%$ & $0.00 \%$ & $100.00 \%$ \\
\hline Financial sector & $0.00 \%$ & $0.00 \%$ & $33.33 \%$ & $66.67 \%$ & $100.00 \%$ \\
\hline Freelancer, Contractor & $0.00 \%$ & $15.63 \%$ & $65.63 \%$ & $18.75 \%$ & $100.00 \%$ \\
\hline Heallhcare & $0.00 \%$ & $20.00 \%$ & $50.00 \%$ & $30.00 \%$ & $100.00 \%$ \\
\hline IT & $16.67 \%$ & $16.67 \%$ & $50.00 \%$ & $16.67 \%$ & $100.00 \%$ \\
\hline Light'haavy industry & $21.43 \%$ & $7.14 \%$ & $57.14 \%$ & $14.29 \%$ & $100.00 \%$ \\
\hline Not specified & $0.00 \%$ & $50.00 \%$ & $50.00 \%$ & $0.00 \%$ & $100.00 \%$ \\
\hline Pensioner & $0.00 \%$ & $0.00 \%$ & $100.00 \%$ & $0.00 \%$ & $100.00 \%$ \\
\hline Physical securty & $0.00 \%$ & $0.00 \%$ & $100.00 \%$ & $0.00 \%$ & $100.00 \%$ \\
\hline Primary sector & $0.00 \%$ & $0.00 \%$ & $100.00 \%$ & $0.00 \%$ & $100.00 \%$ \\
\hline Public administration & $0.00 \%$ & $12.50 \%$ & $37.50 \%$ & $50.00 \%$ & $100.00 \%$ \\
\hline Services & $0.00 \%$ & $33.33 \%$ & $46.67 \%$ & $20.00 \%$ & $100.00 \%$ \\
\hline Student & $20.00 \%$ & $10.00 \%$ & $50.00 \%$ & $20.00 \%$ & $100.00 \%$ \\
\hline Tax consultant & $0.00 \%$ & $0.00 \%$ & $0.00 \%$ & $100.00 \%$ & $100.00 \%$ \\
\hline Transportation & $0.00 \%$ & $0.00 \%$ & $0.00 \%$ & $100.00 \%$ & $100.00 \%$ \\
\hline Celkový soucedet & $5.88 \%$ & $16.91 \%$ & $53.68 \%$ & $23.53 \%$ & $100.00 \%$ \\
\hline
\end{tabular}

Cross analysis: Type of Current Housing and Overall Current Housing Satisfaction

\begin{tabular}{|c|c|c|c|c|c|}
\hline \multirow{2}{*}{$\begin{array}{l}\text { COUNTA of A: CURRENT HOUSING SATISFACTION } \\
\text { CURRENT HOUSING }\end{array}$} & \multicolumn{5}{|c|}{ A: CURRENT HOUSING SATISFACTION } \\
\hline & 2 & 3 & 4 & & ý souçat \\
\hline Apariment - Own & $7.50 \%$ & $20.00 \%$ & $50.00 \%$ & $22.50 \%$ & $100.00 \%$ \\
\hline Apariment - Rent & $12.50 \%$ & $27.50 \%$ & $50.00 \%$ & $10.00 \%$ & $100.00 \%$ \\
\hline Aparment Association - Rent & $0.00 \%$ & $0.00 \%$ & $100.00 \%$ & $0.00 \%$ & $100.00 \%$ \\
\hline Aparlment bulilding - Own (tenement house) & $0.00 \%$ & $40.00 \%$ & $20.00 \%$ & $40.00 \%$ & $100.00 \%$ \\
\hline Apariment outside the city - Own & $0.00 \%$ & $0.00 \%$ & $100.00 \%$ & $0.00 \%$ & $100.00 \%$ \\
\hline Family house - Own (also muligeneratio & $0.00 \%$ & $5.00 \%$ & $55.00 \%$ & $40.00 \%$ & $100.00 \%$ \\
\hline Family house - Rent (also multigenerational) & $0.00 \%$ & $0.00 \%$ & $85.71 \%$ & $14.29 \%$ & $100.00 \%$ \\
\hline Family house multigenerational - villege & $0.00 \%$ & $0.00 \%$ & $100.00 \%$ & $0.00 \%$ & $100.00 \%$ \\
\hline Hut - Cottage - Summer House & $0.00 \%$ & $0.00 \%$ & $100.00 \%$ & $0.00 \%$ & $100.00 \%$ \\
\hline Celkory soucet & $5.88 \%$ & $6.91 \%$ & $53.68 \%$ & $23.53 \%$ & $100.00 \%$ \\
\hline
\end{tabular}

Cross analysis: Location of Current Housing and Overall Current Housing Satisfaction

\begin{tabular}{|c|c|c|c|c|c|}
\hline \multirow{2}{*}{$\begin{array}{l}\text { COUNTA of A: CURRENT HOUSING SATISFACTION } \\
\text { LOCATION OF HOUSING }\end{array}$} & \multicolumn{5}{|c|}{ A: CURRENT HOUSING SATISFACTION } \\
\hline & 2 & 3 & 4 & & ý soucuet \\
\hline Distant surroundings of the canter - former villages, mostly family houses & $0.00 \%$ & $4.76 \%$ & $61.90 \%$ & $33.33 \%$ & $100.00 \%$ \\
\hline Housing estate - collective housing & $4.35 \%$ & $30.43 \%$ & $56.52 \%$ & $8.70 \%$ & $100.00 \%$ \\
\hline Near the city center - mostly multi-storey apartment buldings & $11.54 \%$ & $19.23 \%$ & $48.08 \%$ & $21.15 \%$ & $100.00 \%$ \\
\hline The central part of the cily - mostly mult-storey apartment buildings & $8.33 \%$ & $33.33 \%$ & $25.00 \%$ & $33.33 \%$ & $100.00 \%$ \\
\hline Urban sprawl - individual housing & $0.00 \%$ & $0.00 \%$ & $85.71 \%$ & $14.29 \%$ & $100.00 \%$ \\
\hline Cekovy součet & $5.88 \%$ & $16.91 \%$ & $53.68 \%$ & $23.53 \%$ & $100.00 \%$ \\
\hline
\end{tabular}


IO $^{\text {th }}$ ACAU 2021 conference papers

\section{Figure 5: Correlation analysis 1}

Spearman's correlation: Overall Current Residential Satisfaction and Components of Residential Satisfaction.

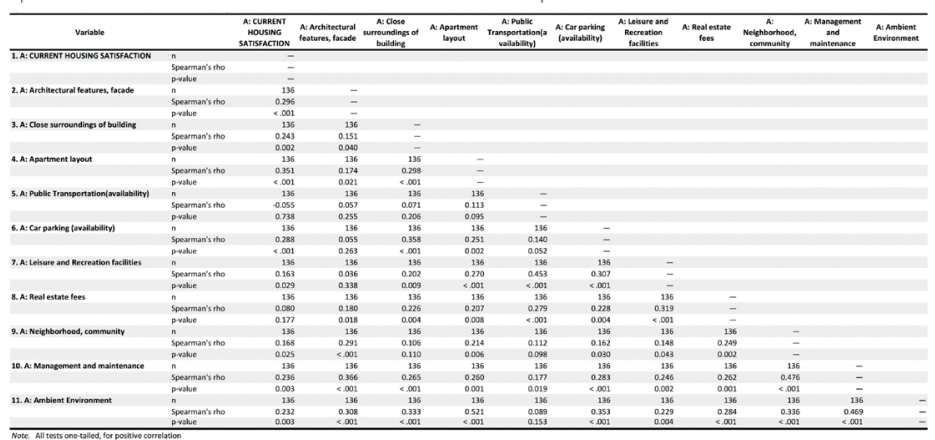

Correlation heatmap: Overall Current Residential Satisfaction and Components of Residential Satisfaction.

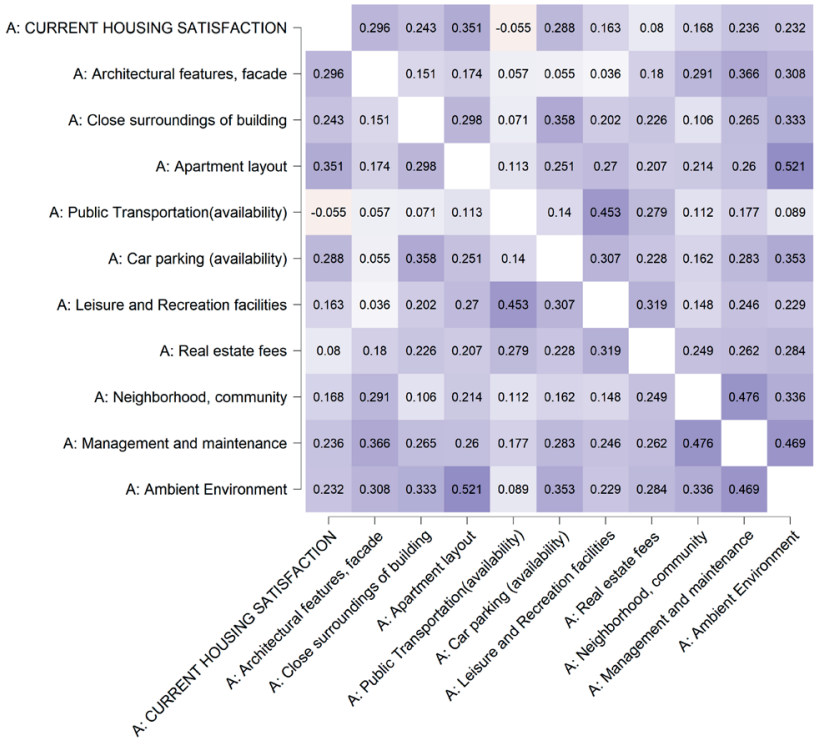




\section{Figure 6: Correlation analysis 2}

Spearman's correlation: Metropolitan House Subj. Evaluation: Overall - Appearance and Facade and Components of Residential Satisfaction

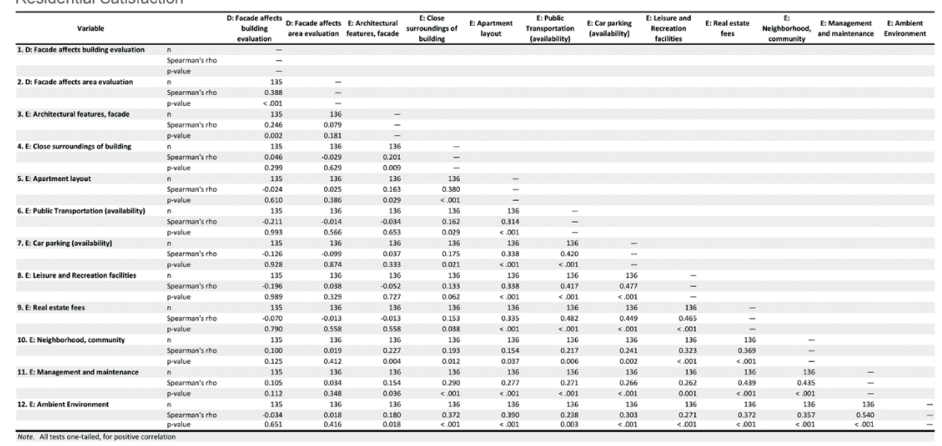

Correlation heatmap: Metropolitan House Subj. Evaluation: Overall - Appearance and Facade and Components of Residential Satisfaction

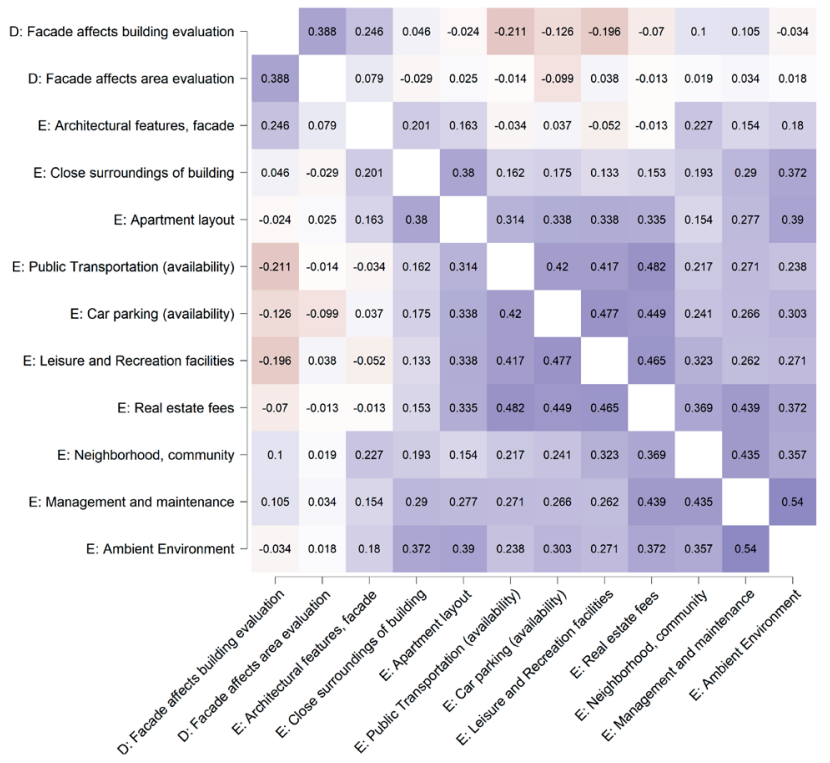


$\mathrm{IO}^{\text {th }}$ ACAU 2021 conference papers

Figure 7: Correlation analysis 3

Spearman's correlation: Metropolitan House Subjective Evaluation: Suitability of the investment and Components of Residential Satisfaction

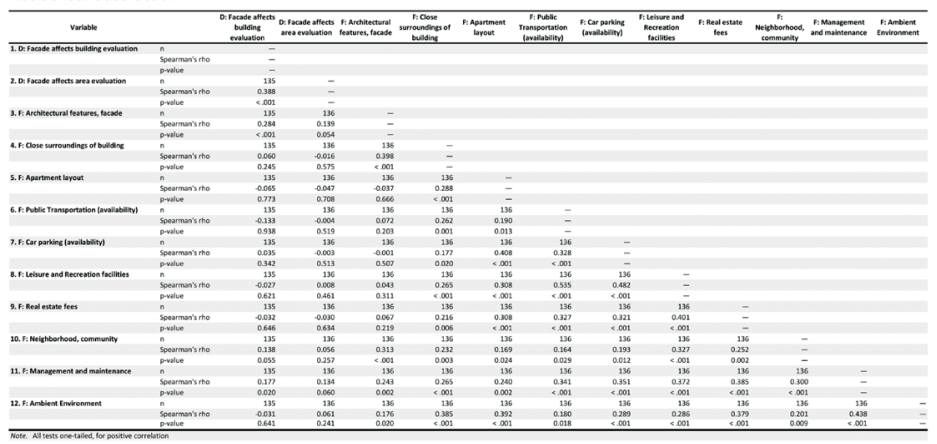

Correlation heatmap: Metropolitan House Subjective Evaluation: Suitability of the investment and Components of Residential Satisfaction

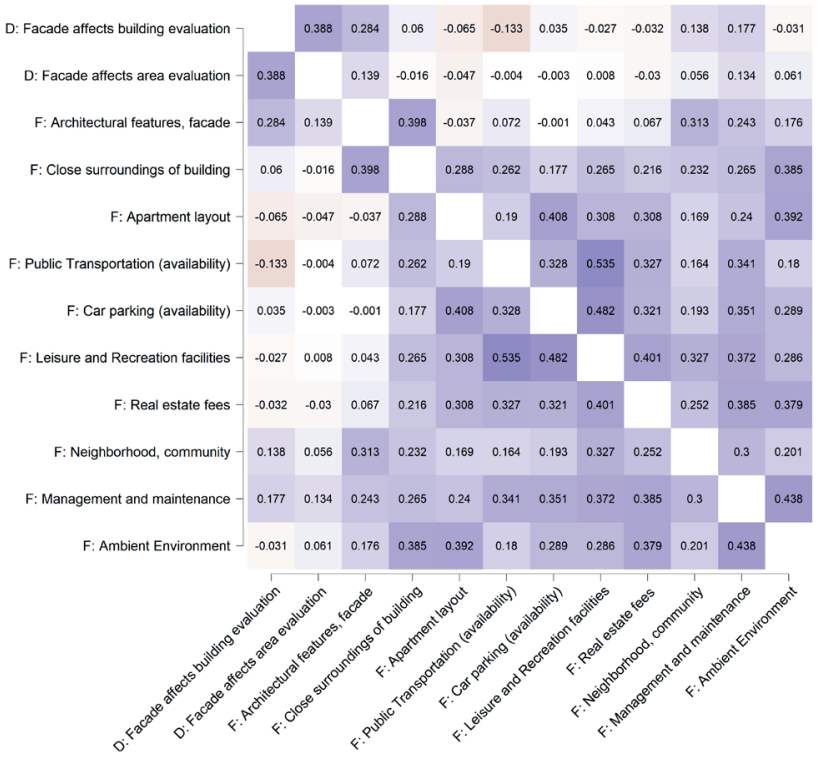


Figure 8: Network analysis 1

Clustering measurers per variable: Overall Current Residential Satisfaction and Components of Residential Satisfaction.

\begin{tabular}{lrrrr}
\hline \multirow{2}{*}{\multicolumn{1}{c}{ Variable }} & \multicolumn{5}{c}{ Network } \\
\cline { 2 - 6 } & Barrat & Onnela & \multicolumn{1}{c}{ WS } & Zhang \\
\hline A: Ambient Environment & -0.576 & 0.030 & -0.669 & -0.562 \\
A: Apartment layout & -0.889 & -0.299 & -0.796 & -1.130 \\
A: Architectural features, facade & 0.332 & 0.108 & 0.267 & 0.381 \\
A: Car parking (availability) & 0.478 & 0.285 & 0.267 & -0.632 \\
A: Close surroundings of building & 0.026 & -0.301 & -0.163 & 0.285 \\
A: CURRENT HOUSING SATISFACTION & -1.178 & -1.049 & -0.796 & -1.096 \\
A: Leisure and Recreation facilities & -0.774 & -0.804 & -0.619 & -0.825 \\
A: Management and maintenance & -0.478 & -0.799 & -0.796 & -0.046 \\
A: Neighborhood, community & 1.750 & 2.223 & 1.861 & 1.716 \\
A: Public Transportation(availability) & 1.750 & 1.355 & 1.861 & 1.706 \\
A: Real estate fees & -0.441 & -0.749 & -0.416 & 0.203 \\
\hline
\end{tabular}

Summary of the network: Overall Current Residential Satisfaction and Components of Residential Satisfaction.

\begin{tabular}{ccc}
\hline Number of nodes & Number of non-zero edges & Sparsity \\
\hline 11 & $31 / 55$ & 0.436
\end{tabular}

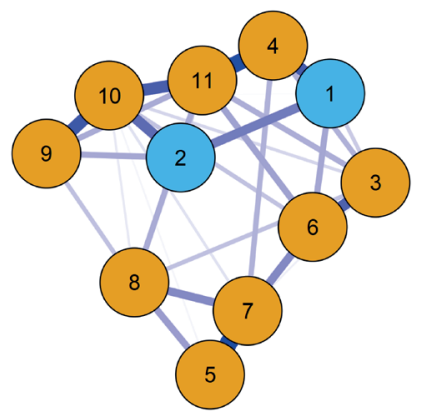

\section{Group 1}

- 3: A: Close surroundings of building

- 4: A: Apartment layout

- 5: A: Public Transportation(availability)

- 6: A: Car parking (availability)

- 7: A: Leisure and Recreation facilities

- 8: A: Real estate fees

- 9: A: Neighborhood, community

- 10: A: Management and maintenance

- 11: A: Ambient Environment

\section{Group 2}

- 1: A: CURRENT HOUSING SATISFACTION

- 2: A: Architectural features, facade 
IO $^{\text {th }}$ ACAU 2021 conference papers

\section{Figure 9: Network analysis 2}

Clustering measurers per variable: Metropolitan House Subj. Evaluation: Overall - Appearance and Facade and Components of Residential Satisfaction

\begin{tabular}{lrrrr}
\hline \multirow{2}{*}{\multicolumn{1}{c}{ Variable }} & \multicolumn{5}{c}{ Network } \\
\cline { 2 - 5 } & Barrat & \multicolumn{1}{c}{ Onnela } & \multicolumn{1}{c}{ WS } & Zhang \\
\hline D: Facade affects area evaluation & -2.029 & -1.939 & -2.287 & -1.652 \\
D: Facade affects building evaluation & -1.841 & -1.660 & -1.841 & -1.564 \\
E: Ambient Environment & 0.550 & 0.446 & 0.472 & 0.031 \\
E: Apartment layout & -0.120 & 0.279 & 0.387 & -0.074 \\
E: Architectural features, facade & -0.560 & -0.831 & -0.058 & -1.258 \\
E: Car parking (availability) & 0.726 & 0.405 & 0.684 & 1.067 \\
E: Close surroundings of building & 0.076 & 0.191 & 0.387 & 0.246 \\
E: Leisure and Recreation facilities & 0.584 & 0.810 & 0.387 & 0.280 \\
E: Management and maintenance & 0.743 & 0.159 & 0.090 & 0.429 \\
E: Neighborhood, community & 0.322 & -0.114 & 0.260 & 0.724 \\
E: Public Transportation (availability) & 1.033 & 1.325 & 0.833 & 1.468 \\
E: Real estate fees & 0.516 & 0.930 & 0.684 & 0.303 \\
\hline
\end{tabular}

Summary of the network: Metropolitan House Subj. Evaluation: Overall - Appearance and Facade and Components of Residential Satisfaction

\begin{tabular}{ccc}
\hline Number of nodes & Number of non-zero edges & Sparsity \\
\hline 12 & $33 / 60$ & 0.500
\end{tabular}

Network analysis: Metropolitan House Subj. Evaluation: Overall - Appearance and Facade and Components of Residential Satisfaction

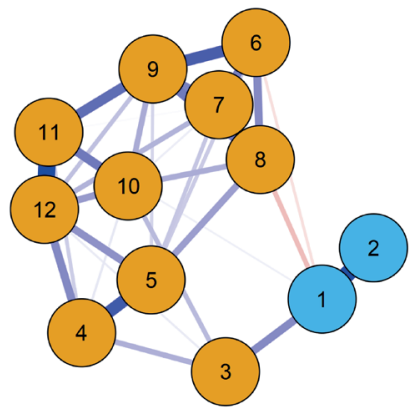

Group 1

- 3: E: Architectural features, facade

- 4: $\mathrm{E}$ : Close surroundings of building

- 5: E: Apartment layout

- 6: E: Public Transportation (availability)

- 7: E: Car parking (availability)

- 8: E: Leisure and Recreation facilities

- 9: E: Real estate fees

- 10: E: Neighborhood, community

- 11: E: Management and maintenance

- 12: E: Ambient Environment

\section{Group 2}

- 1: D: Facade affects building evaluation

- 2: D: Facade affects area evaluation 


\section{Figure 10: Network analysis 3}

Clustering measurers per variable: Metropolitan House Subjective Evaluation: Suitability of the investment and Components of Residential Satisfaction

\begin{tabular}{lrrrr}
\hline \multirow{2}{*}{\multicolumn{1}{c}{ Variable }} & \multicolumn{5}{c}{ Network } \\
\cline { 2 - 5 } & \multicolumn{1}{c}{ Barrat } & Onnela & \multicolumn{1}{c}{ WS } & \multicolumn{1}{c}{ Zhang } \\
\hline D: Facade affects area evaluation & 2.255 & 0.474 & 2.370 & -1.073 \\
D: Facade affects building evaluation & -1.104 & -1.744 & -0.829 & -1.720 \\
F: Ambient Environment & -0.374 & 1.978 & -0.296 & 0.555 \\
F: Apartment layout & -0.951 & -0.885 & -0.931 & -0.075 \\
F: Architectural features, facade & -0.335 & -0.235 & -0.296 & -1.001 \\
F: Car parking (availability) & 0.899 & 1.098 & 1.304 & 0.050 \\
F: Close surroundings of building & -0.936 & -0.639 & -0.829 & -0.153 \\
F: Leisure and Recreation facilities & 0.475 & 0.477 & 0.339 & -0.137 \\
F: Management and maintenance & -1.059 & -1.025 & -1.036 & -0.066 \\
F: Neighborhood, community & 0.351 & 0.148 & 0.237 & 0.329 \\
F: Public Transportation (availability) & 0.594 & 0.112 & -0.118 & 1.592 \\
F: Real estate fees & 0.184 & 0.241 & 0.085 & 1.698 \\
\hline
\end{tabular}

Summary of the network: Metropolitan House Subjective Evaluation: Suitability of the investment and Components of Residential Satisfaction

\begin{tabular}{ccc}
\hline Number of nodes & Number of non-zero edges & Sparsity \\
\hline 12 & $34 / 60$ & 0.485
\end{tabular}

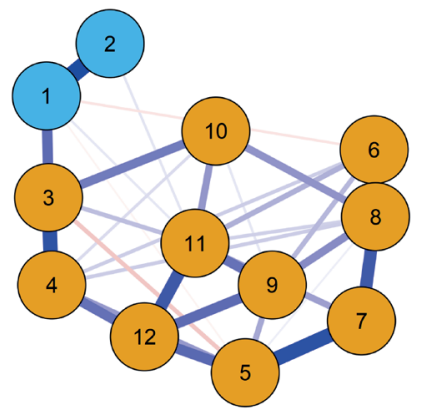

Group 1

- 3: F: Architectural features, facade

- 4: $F$ : Close surroundings of building

- 5: F: Apartment layout

- 6: F: Public Transportation (availability)

- 7: F: Car parking (availability)

- 8: F: Leisure and Recreation facilities

- 9: F: Real estate fees

- 10: F: Neighborhood, community

- 11: F: Management and maintenance

- 12: F: Ambient Environment

Group 2

- 1: D: Facade affects building evaluation

- 2: D: Facade affects area evaluation 